\section{SUITABILITY FOR SUBCUTANEOUS IMPLANTABLE CARDIOVERTER-DEFIBRILLATOR THERAPY - THE IMPACT OF DIFFERENT SELECTION POLICIES}

Amit Kaura*, Nicholas Sunderland, Ravi Kamdar, Ed Petzer, Francis Murgatroyd, Para Dhillon, Paul Scott

\subsection{6/heartjnl-2017-311726.5}

Background With subcutaneous ICDs (S-ICD) not offering the same range of pacing therapies as transvenous systems ( $\mathrm{T}$ ICD), it is unclear how many ICD recipients may be suitable for a S-ICD. In addition, the benefit from ICD therapy is dependent on non-sudden cardiac death (SCD) risk. The study aims were to [1] establish how many ICD recipients may be suitable for S-ICD implantation based on a range of selection policies, and [2] determine the impact of S-ICD use on the risk of non-SCD.

Methods We performed a retrospective study of all ICD implants over 5 years (2009-2013). We evaluated how widely the S-ICD could be utilised using the following indications: (a) Least liberal use - S-ICD used only in patients with inherited channelopathies and idiopathic ventricular fibrillation; (b) Intermediate use - S-ICD also used for SCD primary prevention in hypertrophic cardiomyopathy; (c) Most liberal use - SICD also used for SCD primary prevention in coronary artery disease (CAD) and non-ischaemic dilated cardiomyopathy (DCM) who have a narrow QRS complex (120msec). Given the potential impact of age on the benefit from S-ICDs, we stratified our results by implant age (70, 60 and 50 years). Non-SCD risk was quantified using the MADIT-II risk score (score 0-5, 5 (highest risk)). Mann-Whitney $\mathrm{U}$ testing was used to compare risk scores.

Results Over the 5 years, there were 402 implants (79\% male, age $70 \pm 13$ years). After exclusion of patients implanted with a CRT-D device or with a pacing indication, 219 remained for consideration of a S-ICD. Using our definition of least liberal use, intermediate use, and most liberal use, the number of patients considered suitable for a S-ICD was $18(4.5 \%), 30$ $(7.5 \%)$ and 77 (19.2\%), respectively. Age as a discriminator had a significant impact on the proportion of patients considered suitable for a S-ICD. This was predominantly due to a reduction in the number of patients with CAD and DCM being considered for a prophylactic device. Using the most liberal definition, the number of potential S-ICD candidates dropped from $52(12.9 \%)$ in those 70 years old at implant, to $34(8.5 \%)$ for 60 years, to $20(5 \%)$ using 50 years as the cutoff.

The MADIT-II score was $0.22 \pm 0.1,0.47 \pm 0.1$ and 0.96 \pm 0.1 , in the least liberal, intermediate and most liberal use $S$ ICD groups, respectively. The score was $2.07 \pm 0.1,2.11$ \pm 0.1 and $2.24 \pm 0.1$ in the respective T-ICD groups. The mean MADIT-II score in the S-ICD group was significantly lower than that in the respective T-ICD group $(p<0.0001$, for all three selection policies).

Conclusions While up to one-fifth of patients currently receiving ICDs may be suitable for a S-ICD, this result is dependent on both which patient groups the S-ICD is used in and whether an age cut-off is applied. The cohort of patients suitable for a S-ICD also have a lower risk of non-SCD compared to those receiving conventional T-ICDs.

\section{PLEASE DONT SHOCK ME: A QI PROJECT ON IMPLANTABLE CARDIOVERTER DEFIBRILLATORS (ICDS) AT THE END OF LIFE CARE \& DNAR}

Mahvesh Javaid*, Suzanne Squirrell, Fahad Farooqi. Barking, Havering and Redbridge (BHR) NHS Trust

\subsection{6/heartjnl-2017-311726.52}

Introduction There is an increase in Implantable Cardioverter Defibrillators (ICDs) and Cardiac Resynchronization Therapy Defibrillators (CRT-Ds) being implanted every year. With patients living longer, the number of patients reaching the end of life with an active ICD or CRT-D is also on the rise. These can be missed or be mistaken for a pacemaker, especially during the end of life care.

Problem In 2015, at our hospital, 75\% of patients with an ICD/CRT-D who died had no deactivation. Some of these patients did receive painful and undesirable shocks from their active ICD/CRT-D upon death; unfortunately witnessed by some of their worried families. The hospital staff were surveyed about the awareness on ICDs/CRT-Ds at the end of life and upon death, which revealed $>60 \%$ of staff were unaware what to do.

Aims/Methodology A QI project was established to increase the number of ICD/CRT-D deactivation discussions and switch
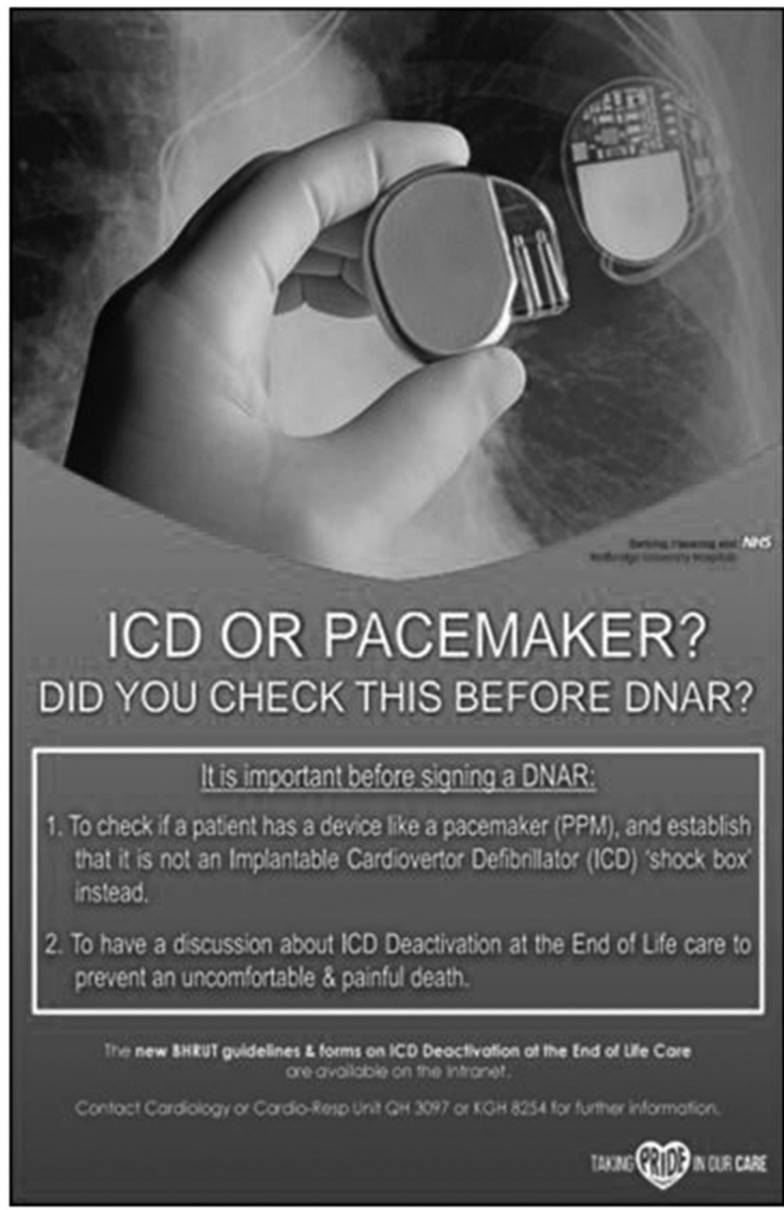

Abstract 53 Figure 1 
offs during the end of life care by 50\%, over a 6 month period. Since January 2016, there were different interventions every month; including Grand round, departmental teaching, posters (Figure 1), implementing related questions in the palliative pathway and e-messages.

Results After 4 months, $66 \%$ of patients with an active ICD/ CRT-D received discussions and switch-offs in advance with good timing before death (Figure 2). In turn, there was a strong impact on patient-centred care, quality and co-ordinated care, efficiency, effectiveness, cost and patient safety.

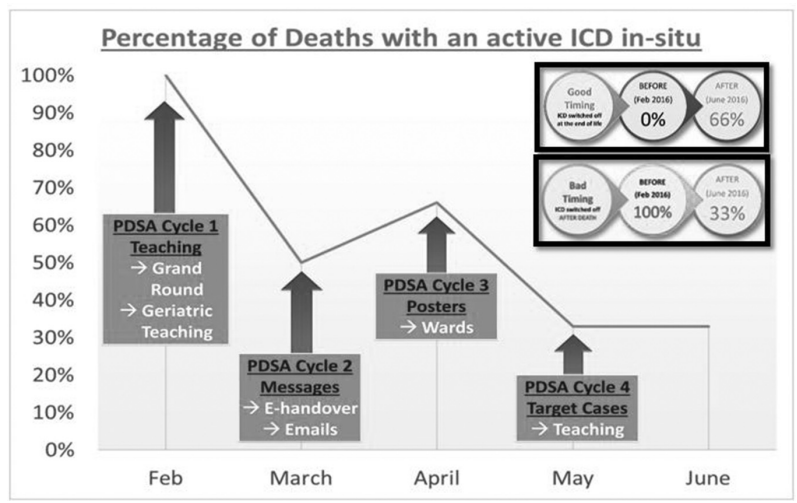

Abstract 53 Figure 2

Conclusions ICDs/CRT-Ds can save lives. However, patients reaching the end of life should be put at ease and comfort by discussing ICD/CRT-D deactivation at DNAR ('Do Not Attempt Resuscitation') discussion and have their ICD/CRT-D switched off in advance with good timing.

\section{Acute Coronary Syndromes}

\section{FRAIL OLDER PATIENTS HAVE A GREATER IMPROVEMENT IN PHYSICAL QUALITY OF LIFE THAN ROBUST PATIENTS AT ONE YEAR FOLLOWING INVASIVE TREATMENT FOR NON-ST ELEVATION ACUTE CORONARY SYNDROME}

${ }^{1}$ Hannah Sinclair, ${ }^{2}$ Sophie Zhaotao Gu, ${ }^{2} J o n a t h a n$ Batty, ${ }^{2}$ Benjamin Beska, ${ }^{1}$ Murugapathy Verrasamy, ${ }^{3}$ Gary Ford, ${ }^{4}$ Weiliang Qiu, ${ }^{2}$ Vijay Kunadian*. ${ }^{1}$ Institute of Cellular Medicine, Newcastle University, and Cardiothoracic Centre, Freeman Hospital; ${ }^{2}$ Institute of Cellular Medicine, Faculty of Medical Sciences, Newcastle University; ${ }^{3}$ Divison of Medical Sciences, Oxford University; ${ }^{4}$ Channing Division of Network Medicine, Department of Medicine, Brigham and Women's Hospital/Harvard Medical School

\subsection{6/heartjnl-2017-311726.53}

Introduction Frailty, a vulnerability to physiological stressors due to a decline in reserve with age, is common in older patients who present with non-ST elevation coronary syndrome (NSTEACS) and is associated with a disproportionately high morbidity and mortality. We aimed to determine how frailty influences physical quality of life (QoL) outcomes after invasive treatment for NSTEACS.

Methods From February 2013 to April 2015, 232 patients aged 75 years with NSTEACS were enrolled into a multicentre prospective observational study. Frailty was assessed using the Fried criteria, where a score of 0 is robust, 1 or 2 is pre-frail and 3 is frail. QoL was evaluated using the Short Form-36 (SF-36) questionnaire (license number QM033917) at baseline and 1 year follow-up. The norm-based Physical Component Score (PCS), an aggregated summary score of the 8 SF-36 subscales, is reported.

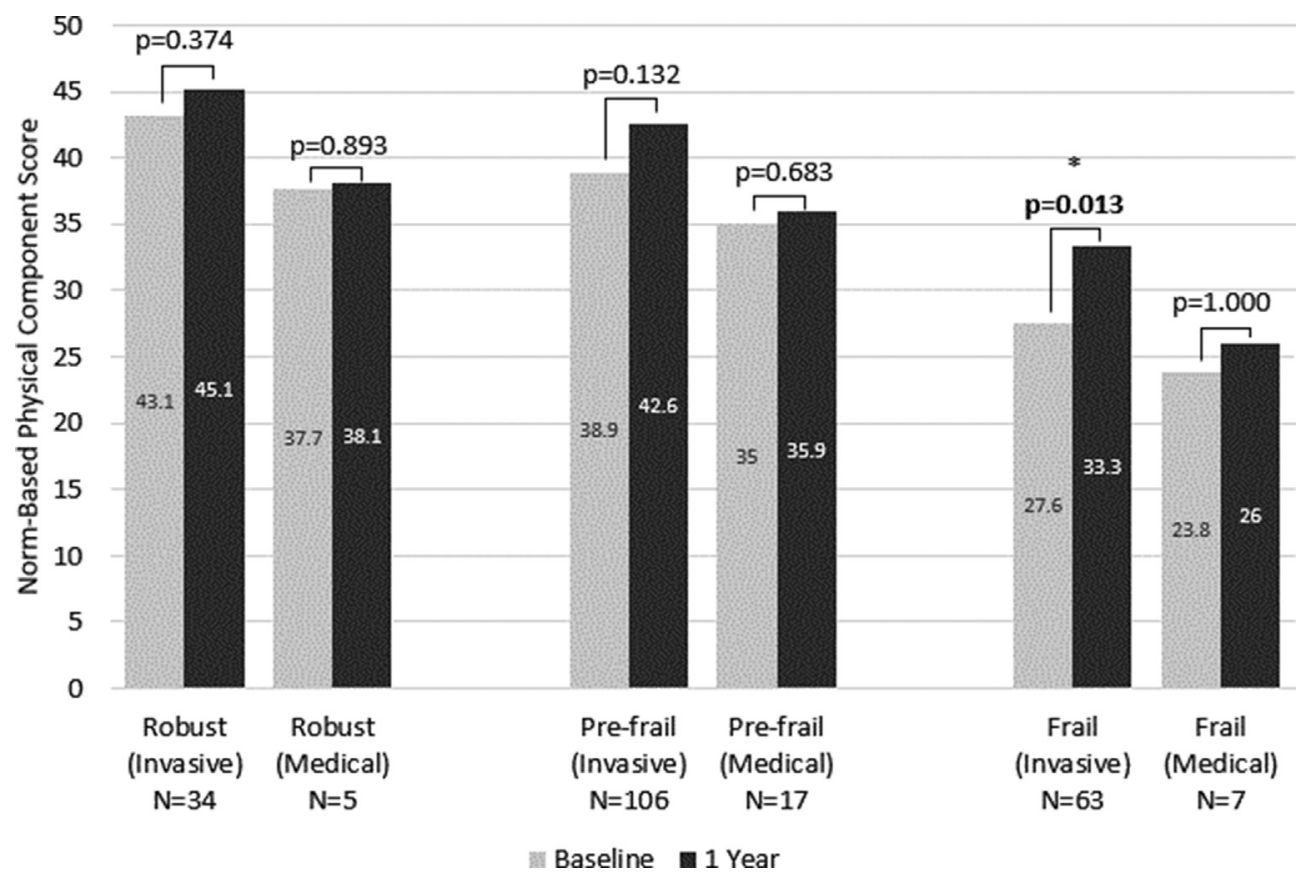

Abstract 54 Figure 1 Change In PCS By Frailty And Treatment 